\title{
From Virus to vector to medicine: Foreword by guest editors
}

\author{
Florian Kreppel $^{1} \cdot$ Anja Ehrhardt ${ }^{2}$
}

Published online: 9 August 2017

(C) Springer Science+Business Media, LLC 2017

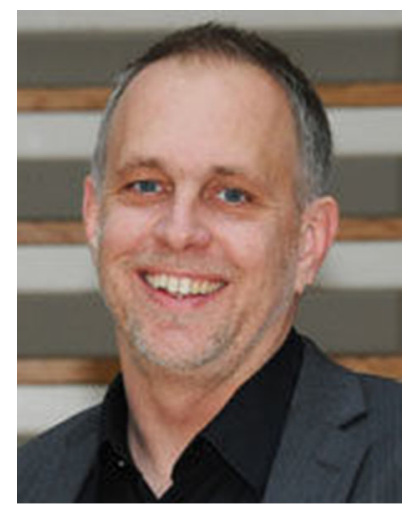

Florian Kreppel

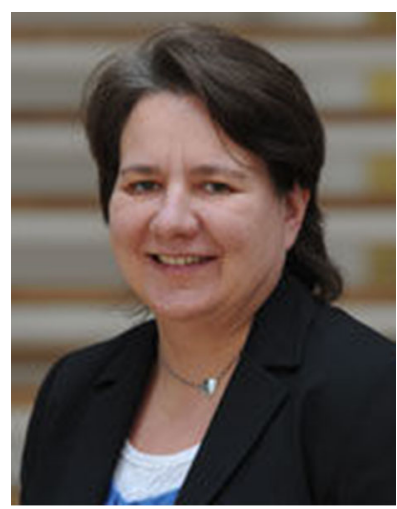

Anja Ehrhardt

We are pleased to publish a Thematic issue of Virus Genes entitled "From virus to vector to medicine." This special study provides an overview of viruses which were utilized as medical products by converting them into vectors (see also Fig. 1). These virus-based vectors are used in gene therapeutic approaches as transport vehicle to insert recombinant therapeutic nucleic acids into the desired

Florian Kreppel

florian.kreppel@uni-wh.de

$\triangle$ Anja Ehrhardt

anja.ehrhardt@uni-wh.de

1 Chair for Biochemistry and Molecular Medicine, Center for Biomedical Education and Research (ZBAF), Department of Human Medicine, Faculty of Health, University Witten/ Herdecke, Witten, Germany

2 Chair for Virology and Microbiology, Center for Biomedical Education and Research (ZBAF), Department of Human Medicine, Faculty of Health, University Witten/Herdecke, Witten, Germany

target cell or as oncolytic agent to treat a broad variety of different diseases. While it had been proposed already long ago, this basic concept represents a modern field of molecular medicine today. Especially during the past decade the field of gene therapy started to thrive and researchers from different disciplines developed novel and innovative treatment options for patients suffering from devastating and often life-threatening diseases. Clinical studies included, for instance, gene therapeutic treatment of patients suffering from severe-combined immune-deficiencies (SCID), hemophilia B, Leber's congenital amauroses (LCA), adrenoleukodystrophy (ALD), Sickle cell disease, and Wiskott Aldrich syndrome (WAS). In 2012, the European Medicines Agency approved the first gene therapeutic drug in Europe: Glybera, a virus vector treating lipoprotein lipase deficiency (LPLD). Up to date more than 2000 gene therapeutic clinical trials worldwide have been performed. Notably, these exciting studies were only rendered possible because ground breaking work in basic virology research as well as translational studies in preclinical in vitro and in vivo models.

Initially, the principle of gene therapy sounded simple because the idea was to utilize genetically modified viruses in which certain viral genes were deleted and replaced by a therapeutic nucleic acid. These recombinant viruses can then be used as vectors to deliver the therapeutic cargo efficiently into affected target cells or unaffected organs that serve as protein production sites. However, especially two clinical trials performed in 1999 and one which was started in 2000 aiming at treating patients suffering from ornithine transcarbamoylase deficiency (OTC) and SCID, respectively, clearly revealed limitations of viruses used as transfer vehicles for gene therapeutic approaches. With these fundamental studies it became clear that the virusbased vector itself was majorly involved in two drawbacks: 


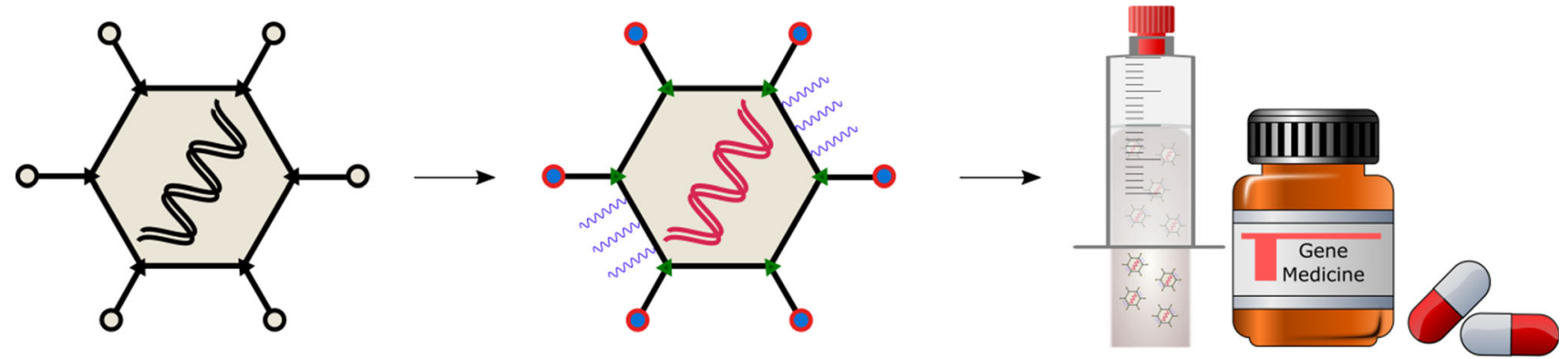

Fig. 1 From virus to vector to medicine. The left panel shows a virus which is converted into a vector (middle panel: green triangles, red circles, and blue dots represent genetically modified capsid/envelope proteins for targeting; red helical molecule represents a modified viral genome including a non-viral therapeutic gene; blue serpentine line represents a chemical capsid/envelope modification for shielding from unwanted vector-host interactions) and then used as advanced medical product in gene medicine that can be applied via different routes (right panel, syringe, pills, and container) (Color figure online)

models [9]. Preclinical studies in large animal models are mandatory to translate basic findings into the clinic. Safety issues are another critical parameter when applying virusbased vectors as advanced medical drugs. Therefore, detailed characterization of produced viral vectors, titrations, and detection are essential, and modern technologies need to be applied. Thus, an additional article [10] describes newest detection methods for viral vectors from the point of view of a regulatory authority. the basic virology of these viruses and their gene functions. Main viruses which are currently used as vectors in gene therapeutic studies are represented by adenoviruses, retroviruses including murine leukemia virus (MLV) and lentiviruses, adeno-associated viruses (AAV), and herpes viruses, but also measles viruses were applied. All transfer vehicles display advantages and disadvantages and only if basic biological features and virus-host interactions of these viruses are investigated, a viral vector can be approved and translated into the clinic as medical drug.

This Thematic issue of Virus Genes addresses exactly this strategy. It will discuss these viruses and their related vectors in detail and provide a state-of-the-art overview of recent applications and techniques in gene therapeutic approaches. Therefore, experts working with different viruses and respective vectors were invited to contribute. Types of viruses which will be discussed include adenovirus [1], AAV [2], retroviral vectors [3], herpes viruses [4]. Applications of the main virus types in gene therapy, oncolytic therapy using adenoviruses [5], immune therapies using lentiviruses [6], and vaccination using measles virus [7] are described. To proof that these gene therapeutic tools are effective, extensive preclinical studies were performed. Especially after systemic administration of virus vectors complex vector-host interactions immediately impose barriers for successful delivery to the target tissue that need to be overcome. This issue is discussed in detail by another article [8]. Furthermore, one article of this issue describes applications of viral vectors in large animal

\section{References}

1. W. Zhang, A. Ehrhardt, Getting genetic access to natural adenovirus genomes to explore vector diversity. Virus Genes (2017). doi:10.1007/s11262-017-1487-2

2. J. Weinmann, D. Grimm, Next-generation AAV vectors for clinical use: an ever-accelerating race. Virus Genes (2017). doi:10.1007/s11262-017-1502-7

3. C. Elsner, J. Bohne, The retroviral vector family: something for everyone. Virus Genes (2017). doi:10.1007/s11262-017-1489-0

4. S.M. Bailer, C. Funk, A. Riedl et al., Herpesviral vectors and their application in oncolytic therapy, vaccination, and gene transfer. Virus Genes (2017). doi:10.1007/s11262-017-1482-7

5. J. Niemann, F. Kühnel, Oncolytic viruses: adenoviruses. Virus Genes (2017). doi:10.1007/s11262-017-1488-1

6. H. Olbrich, C. Slabik, R. Stripecke, Reconstructing the immune system with lentiviral vectors. Virus Genes (2017). doi:10.1007/ s11262-017-1495-2

7. M.D. Mühlebach, Vaccine platform recombinant measles virus. Virus Genes (2017). doi:10.1007/s11262-017-1486-3

8. F. Joensson, F. Kreppel, Barriers to systemic application of virusbased vectors in gene therapy: lessons from adenovirus type 5 . Virus Genes (2017). doi:10.1007/s11262-017-1498-z

9. N. Brunetti-Pierri, P. Ng, Gene therapy with helper-dependent adenoviral vectors: lessons from studies in large animal models. Virus Genes (2017). doi:10.1007/s11262-017-1471-x

10. T. Stellberger, N. Koehler, A. Dinkelmeier et al., Strategies and methods for the detection and identification of viral vectors. Virus Genes (2017). doi:10.1007/s11262-017-1492-5 